\title{
Towards a universal competitive intelligence process model
}

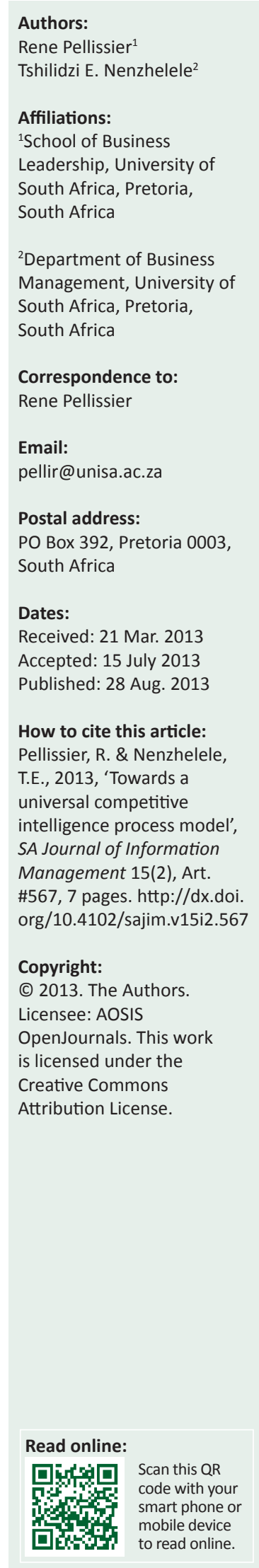

Background: Competitive intelligence (CI) provides actionable intelligence, which provides a competitive edge in enterprises. However, without proper process, it is difficult to develop actionable intelligence. There are disagreements about how the CI process should be structured. For CI professionals to focus on producing actionable intelligence, and to do so with simplicity, they need a common CI process model.

Objectives: The purpose of this research is to review the current literature on CI, to look at the aims of identifying and analysing CI process models, and finally to propose a universal CI process model.

Method: The study was qualitative in nature and content analysis was conducted on all identified sources establishing and analysing CI process models. To identify relevant literature, academic databases and search engines were used. Moreover, a review of references in related studies led to more relevant sources, the references of which were further reviewed and analysed. To ensure reliability, only peer-reviewed articles were used.

Results: The findings reveal that the majority of scholars view the CI process as a cycle of interrelated phases. The output of one phase is the input of the next phase.

Conclusion: The CI process is a cycle of interrelated phases. The output of one phase is the input of the next phase. These phases are influenced by the following factors: decision makers, process and structure, organisational awareness and culture, and feedback.

\section{Introduction}

In a highly competitive business environment, enterprises must be aware of what their competitors are doing (Weiss \& Naylor 2010). Competitive intelligence (CI) has been described as a strategic tool that helps enterprises to be aware of their competitors' behaviours and plans (Haataja 2011). Competitive intelligence produces actionable intelligence that, in turn, helps enterprises in decision-making (Heppes \& Du Toit 2009). Moreover, CI provides a competitive advantage to enterprises (Brody 2008). Competitive intelligence also helps enterprises to improve their performance (Shi 2011). Competitive intelligence evolved from economics, marketing, military theory, information science and strategic management (Muller 2006). It is a profession following a code of ethics developed by the Society of Strategic and Competitive Intelligence Professionals (SCIP). The code of ethics ensures that CI is conducted ethically and legally. Competitive intelligence is a process that consists of a number of steps.

Whilst the objectives of CI are clear, there is some confusion about how the CI process should be structured (Nasri 2011). Some scholars view CI process as a cycle, whilst others view it as a linear process (Bartes 2012; Cucui 2009). Some scholars outline many stages in the CI process, whilst others identify fewer stages (Nasri 2011). As Du Toit and Muller (2004), Venter and Tustin (2009), Nasri (2011) and Bartes (2012) caution, without a proper process and structure, it is difficult to develop CI. Hence, there is a need for a common understanding of the CI process.

\section{Evolution of competitive intelligence}

Competitive intelligence evolved from economics, marketing, military theory, information science and strategic management (Muller 2006). Competitive intelligence was characterised as being more focused on gathering information than on analysis (Cucui 2009). Moreover, there was very a weak connection between CI and the decision-making process. The focus was on developing information-gathering skills in CI professionals. According to Cucui (2009), analysis of the gathered information began in the 1980s. During this period, SCIP was established with the aim to ensure that CI is conducted in a professional manner. In the late 1980s, the connection between $\mathrm{CI}$ and decision-making was made. During this period, the competitive intelligence review was established (Cucui 2009). Since then, many universities offer courses in CI across the world. 
Enterprises have CI units that analyse collected information professionally. According to Muller (2005), CI took root in South Africa in the mid-1990s and early 2000s. Competitive intelligence is evolving in complexity and importance to maintain pace with rapid business development (Heppes \& $\mathrm{Du}$ Toit 2009). Since the end of the Cold War, CI - once widely used in the military environment - has rapidly infiltrated into business competition (Deng \& Luo 2010).

\section{Definition of competitive intelligence}

There are many definitions of CI in the literature (Oubrich 2011; Weiss \& Naylor 2010). Most of the definitions that have emerged over the years differ only in terms of semantics and emphasis (Fleisher \& Wright 2009). Wright, Eid and Fleisher (2009) support this, stating that there has been tweaking of previous definitions, leaving out one word, adding another, but rarely anything more substantial. Brody (2008) concludes that, because $\mathrm{CI}$ is a process that is set in situations that are dynamic and in which the players are moving forward in a constantly changing business environment, the variety of definitions may be a reflection of that process of constant change. Fleisher and Wright (2009) argue that CI practitioners rarely have time for definitions, but are keen to understand how they can do their job better. For the purposes of this study, Brody's (2008) definition will be adopted because it is broad and simple. Brody (2008) defines CI as:

the process by which enterprises gather actionable information about competitors and the competitive environment and, ideally, apply it to their planning processes and decision-making in order to improve their enterprise's performance. (n.p.)

\section{Competitive intelligence objectives}

The main objectives of competitive intelligence are to provide help in decision-making and to provide an enterprise with a competitive advantage. Competitive intelligence is a way to alert enterprises constantly of changes in the competitive environment (Muller 2005). Researchers have identified the following objectives of CI (Cucui 2009; Peltoniemi \& Vuori 2008; Wright et al. 2009): enhancing the enterprise's competitiveness; predicting, with a high level of trust, the business environment's evolutions, competitors' actions, customers' requirements and even influences generated by political change; providing better support for the strategic decision-making process; revealing opportunities and threats by surveying weak signals and early warnings; processing and combining data and information to produce knowledge and insights about competitors; satisfying the information needs of decision-making and problem-solving, and decreasing reaction time; and devising marketing strategies.

\section{Competitive intelligence process}

Competitive intelligence is a process consisting of phases that are linked (Nasri 2011). The output of each phase is the input to the next phase (Bartes 2012). The overall output of the $\mathrm{CI}$ process is an input to the decision-making process (Wright et al. 2009). Most CI definitions clearly reveal that it is a process that produces actionable intelligence (Brody
2008). According to Du Toit and Muller (2004), without a proper intelligence process and structure, it is difficult to develop intelligence. Also, without the visible support of and utilisation of intelligence by top management, the process will be flawed (Nasri 2011). Put differently, the overriding influence on successful CI process is the existence of a management support, culture and structure that encourages and develops CI activities in companies (Nasri 2011). Therefore, management must plan, support and implement a CI process.

Given the confusion in the field of CI on how the CI process should be structured, some agreement within the CI field on this should be reached (Wright \& Calof 2006). A study conducted by Carr (2003) discovered that CI experts describe the CI process as a cycle, as a linear process, using fourpoint models, as a scientific method and as a pyramid. Some scholars outline many phases in the CI process, whilst others identify fewer phases. Some scholars name the same phases differently, thereby adding to the confusion in the field of CI.

The following CI process models were established in the literature.

According to Calof (1998), the CI process is made up of obtaining a CI request, collecting information, analysing and synthesising information, communicating intelligence, and managing the CI process. This CI process model does not incorporate the capturing and storing of collected information.

Calof and Skinner (1998) view the CI process as a cycle made up of four phases: planning and direction, data collection, information analysis and intelligence dissemination. These two scholars term the information collection phase 'data collection' and omit information capturing and storing. Their CI process model does not incorporate influential factors such as decision-makers, feedback, organisational awareness and culture, and process and structure.

Kahaner (1998) also defines CI as a cycle process with four phases: planning and direction, data and information collection, analysis and dissemination of intelligence to those who will use it. This CI process model omits information capturing and storage and terms the information collection phase 'data and information collection' phase. Information consists of organised data. Therefore, in information there is data; there is no need to use both terms together in the name of this phase.

Melo and Medeiros (2007) add evaluation to Kahaner's (1998) CI process cycle to make it a five-phase cycle composed of planning, collection, analysis, dissemination and evaluation. These scholars also omitted information capturing and storage and the influential factors.

Cruywagen (2002) views the CI process as a cycle with a number of distinguishable phases, including planning and direction, collection, evaluation, analysis and dissemination. 
Although Cruywagen (2002) incorporated feedback, the other influential factors such as decision-makers, organisational awareness and culture and process and structure are omitted. The information capturing and storage phase is also omitted.

Dishman and Calof (2002) establish six phases of the CI process: planning and focus, collection, analysis, communication, process or structure and organisational awareness and culture. Although this is an improved CI process model, it omits information capturing and storage and feedback. According to Viviers, Saayman and Muller (2005), the CI process is a cycle made up of planning and focus; collection; analysis; communication; and awareness, culture, process and structure. This CI process model also omits information capturing and storage and feedback.

Botha and Boon (2008) view the CI process as a cycle consisting of seven phases: intelligence needs and determining key intelligence topics; planning and direction; collection; information processing; analysis; dissemination; and intelligence users and decision-makers. This model incorporates influential factors as phases and omits feedback. However, unlike other scholars, they recognise the need to capture and store collected information.

Wright and Calof (2006) identify four phases of the CI process: planning or focus, collection, analysis and communication. They also indicate that process, structure, culture, awareness and attitude are undeniable influences of CI process success. Their CI process model omits information capturing and storage, decision-makers and feedback.

According to SCIP (2007), CI is a cycle with five phases: planning and direction, collection activities, analysis, dissemination and feedback. This CI process model omits information capturing and storage and other influential factors such as decision-makers, organisational awareness and culture, and process and structure.

Bose (2008) views the CI process as a cycle made up of planning and direction, collection, analysis, dissemination and feedback. This CI process model omits information capturing and storage and other influential factors such as decision-makers, organisational awareness and culture and process and structure.

According to Sawka and Hohhof (2008), the CI process is a cycle made up of the following interrelated phases: planning and direction, collection, analysis and production and dissemination. These scholars term the information analysis phase 'analysis and production'. This means that intelligence is produced in the analysing phase. Their CI process model omits information capturing and storage and all the influential factors previously mentioned.

According to Cucui (2009), CI is a process consisting of the following steps: monitoring business environment, gathering, analysing and filtering and disseminating intelligence. This model differs from the rest of the scholars' models because of the phase names. The planning and direction phase is called 'monitoring business environment'. The information collection phase is called 'gathering' and the intelligence dissemination phase is called 'filtering and dissemination'. This CI process model also omits information capturing and storage and other influential factors mentioned.

Competitive intelligence, according to Shi, Mou and Wan (2009), is a cycle process made up of defining CI demand, gathering information, processing information and providing final services to meet the demand. Just like Cucui (2009), Shi et al. (2009) name their CI process phases differently. They omit information capturing and storage and all the influential factors.

According to Haddadi, Dousset and Berrada (2010), CI is a cycle process made up of understanding the need, researching and gathering information, processing information and disseminating information. These scholars use different phase names and omit information capturing and storage. They also omit all the influential factors and call the information analysis phase 'processing information'.

Muller (2002) identifies six phases in the CI process: planning and focus; collection; analysis; communication; process and structure and organisational awareness and culture. Strauss and Du Toit (2010) propose a seventh phase: 'skills development'. According to them, training clears up misconceptions regarding CI, improves communication, encourages easy transfer of expertise and skills and fosters a mindset of awareness within the enterprise. They conclude that the CI process is not complete without skills development. Their CI process model omits information capturing and storage and feedback.

The CI process, according to McGonagle and Vella (2012), is divided into five phases, each linked to the other by a feedback loop. These phases are: establishing the CI needs, collecting the raw data, evaluating and analysing the raw data, communicating the finished intelligence and taking action. Unlike most scholars, McGonagle and Vella's (2012) CI process model emphasises collection and analysis of data rather than information. Their model introduces the taking action phase, in which decision makers make decisions. They omit other influential factors such as organisational awareness and culture and process and structure.

\section{Research results}

From the CI process models discussed above, the following common and unique phases and characteristics were identified:

\section{Cycle}

The CI process is presented in a cycle of phases. The reason behind the use of a cycle is that the CI process never stops, but is continuous. Also, the cycle is used to indicate that the phases are interrelated. Therefore, the output of one phase 
is the input of the next phase. All scholars in this study represent the $\mathrm{CI}$ process as a cycle.

\section{Establishing competitive intelligence needs}

Some scholars identify this as the first phase of the CI process. Different names are given to this phase, such as 'obtaining CI request', 'intelligence needs and determine key intelligence topics', 'understanding the need', and 'defining CI demand'. According to Botha and Boon (2008), this phase involves identification of intelligence needs of decision-makers and narrowing these intelligence needs down to key intelligence topics (KITs). The KITs are those topics identified as being of greatest significance to an organisation's senior executives, providing purpose and direction for CI operations (Bose 2008). In this phase, the CI director identifies and prioritises both senior management and organisational key intelligence needs. Moreover, what the CI unit should research and to whom this intelligence should be delivered are determined in this phase (Strauss \& Du Toit 2010).

\section{Planning and direction}

Some scholars call this phase 'planning and focus'. In some scholars' CI process models, this is the first phase, whilst it is the second in others. This phase defines the decision-makers' intelligence requirements. It requires knowledge of KITs. The KITs must be clear and not ambiguous. The intelligence requirements must be transformed into information requirements in order to determine if the required information already exists or not. The steps to acquire the required information must be clearly outlined (Nasri 2011).

\section{Information collection}

To some scholars, this is the second phase of the CI process, whilst it is the third phase in other models. This phase is referred to in different ways, such as 'data and information collection', 'collecting raw data', 'researching and gathering information', 'data collection', 'collection', 'gathering' and 'monitoring business environment'. The emphasis is on collection of publicly available information (Botha \& Boon 2008). This is to ensure compliance with the code of ethics developed by SCIP. The information to be collected must be relevant to the KITs. Some common primary sources include government agencies, employees, suppliers, customers and conferences. Some common secondary sources include magazines, TV, radio, analyst reports and professional reports. Information can be collected through Internet searches, surveys, interviews, observation, media scanning and networking (Nasri 2011).

\section{Information processing}

This phase organises, systematises, implements and maintains a mechanism of capturing and storing information. Collected information is sorted and stored in a database. Information stored in electronic format is easy to analyse and disseminate (Nikolaos \& Evangelia 2012).

\section{Information analysis}

Some scholars call this phase 'analysis' or 'analysis and production'. This is the core phase of the CI process (Viviers et al. 2005) and is the most challenging (Nikolaos \& Evangelia 2012). Processed information must be interpreted and analysed to produce actionable intelligence. The analysis methods mostly used include PEST (political or legal, economical, socio-cultural and technological) analysis, scenario analysis, Porter's five forces model, SWOT (strengths, weaknesses, opportunities and threats) analysis and competitor profiling (Viviers et al. 2005).

\section{Intelligence dissemination}

Actionable intelligence is disseminated to decision-makers in this phase. Some scholars call this phase 'communication', 'intelligence dissemination', 'disseminating information', 'communicating the finished intelligence' and 'filtering and disseminating intelligence'. The finished product, which is actionable intelligence, is communicated back to the decision-makers in a format that is easily understood. The communication is in the form of a report, dashboard or meeting. Face-to-face, email and intranet communication are also used (Nasri 2011).

\section{Taking action}

This phase is also called intelligence users and decisionmakers'. In this phase, the decision-makers use actionable intelligence to make decisions (McGonagle \& Vella 2012). This phase leads to the identification of new intelligence needs by users of intelligence and decision-makers and the intelligence cycle or process is activated again (Botha \& Boon 2008).

\section{Skills development}

In this phase, CI professionals are trained on how to conduct their different responsibilities. They get training on interpreting KITs, information collection, information analysis and intelligence dissemination.

\section{Process and structure}

Competitive intelligence requires appropriate policies and procedures, and a formal or informal infrastructure so that employees can contribute effectively to the CI system as well as gain benefits from the process. A CI code of ethics must also be incorporated in CI policies. The CI process depends on gathering people and resources from a range of internal units and encouraging employees to contribute to using and participating in the CI activities (Kahaner 1998).

\section{Organisational awareness and culture}

For CI to flourish in a company and for the discipline to be implemented and used optimally, there has to be an appropriate organisational awareness of CI and a culture of competitiveness. It is important to create the right environment for CI. It requires continuous staff training with emphasis on the importance of CI. Without proper 
awareness and attitudes that favour both intelligence and information sharing, it is difficult to develop intelligence within an organisation (Viviers et al. 2005).

\section{Feedback}

Feedback about the CI process is provided in this phase. This phase is also called 'evaluation'. This phase outlines the feedback from decision-makers and CI professionals (Frion 2009). Feedback and updates from CI professionals allow for midcourse adjustments and new issues to surface (Prescott 1999). The feedback provides opportunities for revisions of the original intelligence request as well as constructive feedback on the deliverable of previous requests facilitating a continuous improvement atmosphere (Nasri 2011). The CI process is improved through feedback (Oubrich 2011).

The frequencies of the phases and characteristics in CI process models are shown in Table 1 below. The table indicates that all scholars in this study view the CI process as a cycle made up of interrelated phases. Also, all scholars in this study have information collection and intelligence dissemination phases in their CI process models. The majority of the scholars have information analysis (89\%) and planning and direction $(78 \%)$ phases in their CI process models. A lesser percentage of the scholars $(28 \%)$ have the following phases in their CI process models: establishing CI needs; process and structure; organisational awareness and culture and feedback. Only $17 \%$ of scholars had an 'information processing' phase in their CI process model. Also, only $11 \%$ of scholars had a 'taking action' phase, whereas only $6 \%$ of scholars had a skills development phase in their CI process model.

The extensive review of the literature and analysis of the above frequencies led to the formulation of a universal $\mathrm{CI}$ process model, as shown in Figure 1. The purposes of each phase of the CI process were thoroughly reviewed and analysed. This CI process model is a cycle because $100 \%$ of the scholars in the literature review consider the CI process to be a cycle. Phases that were called by different names, but which have the same purpose, were merged; for example, the planning and direction phase and the establishing CI needs phase were merged because they serve the same purpose. The phase is now called 'planning and direction' and defines the decision-makers' intelligence requirements.

TABLE 1: Characteristics and phases of competitive intelligence process models.

\begin{tabular}{lll}
\hline Characteristics & $\boldsymbol{f}$ & $\mathbf{\%}$ \\
\hline Cycle & 18 & 100 \\
Establishing Cl needs & 5 & 28 \\
Planning and direction & 14 & 78 \\
Information collection & 18 & 100 \\
Information processing & 3 & 17 \\
Information analysis & 16 & 89 \\
Intelligence dissemination & 18 & 100 \\
Taking action & 2 & 11 \\
Skills development & 1 & 6 \\
Process and structure & 5 & 28 \\
Organisational awareness and culture & 5 & 28 \\
Feedback & 5 & 28 \\
\hline
\end{tabular}

$f$, frequency.
The proposed CI process model has an 'information collection' phase because all CI scholars in the above analysis deemed it necessary. During the information collection phase, information relevant to the KITs is collected legally and ethically from different sources. Most scholars made the assumption that collected information will automatically be sorted, captured and stored. As a result, only a few scholars had information processing as a phase in their CI process models. Although some indicated that information processing involves sorting, capturing and storing collected information, some mistook 'information processing' for 'information analysis'. Without information sorting, capturing and storing, there is a gap in the CI process. Therefore, the proposed CI process model has separated the information processing and information analysis phases. To clear up the confusion, the phase of information processing is called 'information sorting, capturing and sorting' in the proposed CI process model, and is self-explanatory.

Almost all the previous scholars had 'information analysis' as a phase in their CI process models. As a result, the proposed CI process model incorporates an information analysis phase. In this phase, stored information is analysed to produce actionable intelligence. Because all previous scholars incorporated intelligence dissemination in their CI process model, the proposed CI process model has a 'intelligence dissemination' phase. During this phase actionable intelligence is disseminated to decision-makers. Decision-makers use the actionable intelligence to make business decisions. Therefore, there is no need to have a separate 'taking action' phase.

'Skills development' is an inherent part of 'organisational awareness and culture', so the proposed CI process model does not have this as a separate phase. The proposed CI process model is a cycle with influential factors affecting it. Most scholars consider decision-makers; process and structure; organisational awareness and culture and feedback to influence the CI process. CI professionals must consider these factors throughout the CI process. Decision-makers must be contacted in case their inputs are required during the CI process. There must be feedback throughout the CI process phases. All organisational processes and structure that might affect the CI process must be considered throughout the CI process. There has to be an appropriate organisational awareness of $\mathrm{CI}$ and a culture of competitiveness. The smaller circle dips into the larger cycle to indicate that all phases are affected by the influential factors. The proposed CI process model is all encompassing, considering the extensive review of literature.

\section{Discussion}

There are many CI process models described in the literature. Most of these models differ from one another because scholars use different names for the same phases. Some scholars just add or subtract phases. This has led to a confused CI field. 


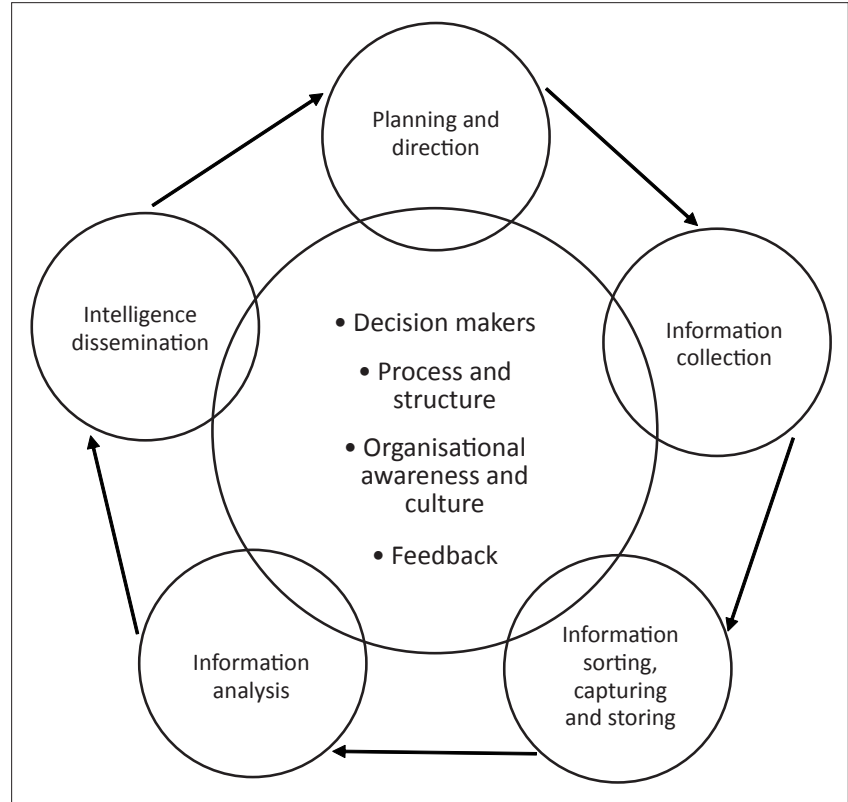

FIGURE 1: Competitive intelligence process model.

There was therefore a need to propose a universal CI process model.

The findings of this study reveal that all scholars view the CI process as a cycle of interrelated phases. This means that the output of one phase is the input of the next phase. Also, it means that the CI process is continuous and does not stop. All scholars have an information collection phase in their CI process models, as well as an intelligence dissemination phase. They realise that intelligence is used by decisionmakers.

The findings reveal that the majority of scholars have information analysis in their CI process models. They acknowledge that information analysis is the core phase of the CI process. They realise that information is useless when it is not interpreted. However, there was some confusion between the information analysis and information processing phases. Those that had one of these phases did not necessarily have the other. Although some indicated that information processing involves the sorting, capturing and storing of collected information, some mistook information process for information analysis. Without information sorting, capturing and storing, there is a gap in the CI process.

Also, the majority of the scholars have a planning and direction phase in their CI process models. They realise that planning is important in a successful CI process. However, there was some confusion between the planning and direction phase and the establishing of CI needs phases. Those scholars who had a planning and direction phase indicated that establishing CI needs is done in this phase. Those who had the establishing CI needs phase indicated that this phase involves the establishment of CI needs and hardly mentioned anything about planning and direction.
The findings also reveal that fewer scholars incorporated process and structure, organisational awareness and culture and feedback into their CI process models. Perhaps this is because these are crucial influences on the CI process. Also, the findings show that feedback should be conducted throughout the CI process and not only at the end of the process. The findings also reveal that fewer scholars had taking action and skills development phases. Perhaps this is because there is an assumption that once the intelligence lands in the hands of decision-makers they will make decisions.

\section{Conclusion}

Without a proper process, it is difficult to develop CI (Du Toit \& Muller 2004). There is disagreement in the CI field with regard to the $\mathrm{CI}$ process. Different names have been given to the phases in the CI process. There is also disagreement as to the number of phases in the various models. According to the model proposed here, establishing CI needs, planning and direction take place in the phase called 'planning and direction'. Influential factors, such as process and structure; organisational awareness and culture; feedback and decision-makers influence all the phases of the CI process. The proposed CI process model incorporates an information sorting, capturing and storing phase. Most scholars did not highlight the sorting, capturing and storing of information phase; an assumption was merely made that collected information would be sorted, captured and stored somehow. The suggested name for this phase, 'information sorting, capturing and storing', is intended to eliminate confusion between the information processing and the information analysis phases.

The proposed CI process model is a cycle, indicating that the process is continuous. It also indicates that the output of one phase is the input for the next stage. Decision-makers take actions after receiving actionable intelligence. Therefore, there is no need to have a separate phase called 'taking action'. Also, because skills development is a part of organisational awareness and culture, there is no need to have a separate 'skills development' item. The proposed CI process model above is comprehensive. This implies that implementing the proposed CI process model will simplify the CI process and ensure that all CI stakeholders will focus on what matters, namely producing actionable intelligence.

\section{Acknowledgement}

The authors would like to acknowledge the University of South Africa for the funding and support without which this research would not have been possible.

\section{Competing interest}

The authors declare that they have no financial or personal relationship(s) that may have inappropriately influenced them in writing this article. 


\section{Authors' contributions}

T.E.N. (University of South Africa) initiated this research, conducted the literature review and wrote the analysis and conclusion. R.P. (University of South Africa) led and supervised this project, selected the journal and handled editorial matters.

\section{References}

Bartes, F., 2012, 'Increasing the competitiveness of company by competitive intelligence', paper presented at the 7th International Scientific Conference intelligence, paper presented at the 7th Internationa
Business and Management, Vilnius, Lithuania, 10-11 May.

Bose, R., 2008, 'Competitive intelligence process and tools for intelligence analysis', Industrial Management \& Data Systems 108(4), 510-528. http://dx.doi. org/10.1108/02635570810868362

Botha, D.F. \& Boon, J.A., 2008, 'Competitive intelligence in support of strategic training and learning', South African Journal of Information Management 10(3), 1-6.

Brody, R., 2008, 'Issues in defining competitive intelligence: An exploration', Journal of Competitive Intelligence and Management 4(3), 3-16.

Calof, J., 1998, 'Increasing your CIQ - the competitive intelligence edge', The Economic Development Journal of Canada, viewed 13 January 2013, from www. ecdevjournal.com/pubs/1998/art022_98.htm

Calof, J.L. \& Skinner, B., 1998, 'Competitive intelligence for government officers: a brave new world', Optimum 28(2), 38-42.

Dishman, P. \& Calof, J.L., 2002, The intelligence process: front-end to strategic planning, University of Ottawa, Ottawa.

Carr, M.M., 2003, Super searchers on competitive intelligence, Reva Basch, New Jersey.

Cruywagen, A., 2002, 'Establishing the profile of a successful competitive intelligence practitioner', paper presented at the Competitive Intelligence World 2002 Conference, The Conference Park, Midrand, Johannesburg, South Africa, 20 November.

Cucui, A.P.G., 2009, 'A framework for enhancing competitive intelligence capabilities using decision support system based on web mining techniques', International Journal of Computers, Communications and Control 4(4), 326-334.

Deng, Z. \& Luo, L., 2010, 'An exploratory discussion of new ways for competitive intelligence on Web2.0', International Federation for Information Processing 252(1), 597-604.

Du Toit, A. \& Muller, M.L., 2004, 'Organizational structure of competitive intelligence activities: a South African case study', South African Journal of Information Management 6(3), Art. \#308, 13 pages.

Fleisher, C.S. \& Wright, S., 2009, 'Examining differences in competitive intelligence practice: China, Japan, and the West', Thunderbird International Business Review 51(3), 249-261. http://dx.doi.org/10.1002/tie.20263

Frion, P., 2009, 'What information behaviour can offer to competitive intelligence', paper presented at the International Symposium Models, Methods, Engineering of Competitive intelligence, Beaulieu-sur-Mer, France, 25-26 November

Haataja, J., 2011, Social media as a source of competitive intelligence in a pharmaceutical corporation, published master's thesis, Degree Programme in Industrial Engineering and Management, School of Science, Aalto University, Helsinki.

Haddadi, A.E., Dousset, B. \& Berrada, I., 2010, Xplor Every Where - The Competitive Intelligence System for Mobile, viewed 9 July 2013, from http://ieeexplore.ieee. org/stamp/stamp.jsp?tp=\&arnumber $=5945635$
Heppes, D. \& Du Toit, A., 2009, 'Level of maturity of the competitive intelligence function: Case study of a retail bank in South Africa', Aslib Proceedings: New Information Perspectives 61(1), 48-66.

Kahaner, L., 1998, Competitive intelligence: how to gather, analyze and use information to move your business to the top, Touchstone, New York.

McGonagle, J.J. \& Vella, C.M., 2012, Proactive Intelligence, Springer-Verlag, London. http://dx.doi.org/10.1007/978-1-4471-2742-0

Melo, M.A.N \& Medeiros, D.D., 2007, 'A model for analyzing the competitive strategy of health plan insurers using a system of competitive intelligence', The TQM Magazine 19(3), 206-216. http://dx.doi.org/10.1108/09544780710745630

Muller, M.L., 2002, Managing competitive intelligence, Knowres Publishing, Johannesburg.

Muller, M.L., 2005, 'Beyond competitive intelligence-innovation and competitive strategy', South African Journal of Information Management 7(1), Art. \#244, 6 pages.

Muller, M.L., 2006, 'Parts of competitive intelligence: competitor intelligence', South African Journal of Information Management 8(1), Art. \#209, 5 pages.

Nasri, W., 2011, 'Competitive intelligence in Tunisian companies', Journa of Enterprise Information Management 24(1), 53-67. http://dx.doi. org/10.1108/17410391111097429

Nikolaos, T. \& Evangelia, F., 2012, 'Competitive intelligence: concept, context and a case of its application', Science Journal of Business Management 2012(2), 1-15.

Oubrich, M., 2011, 'Competitive Intelligence and Knowledge Creation - Outward insights from an empirical survey', Journal of Intelligence Studies in Business 1(1), 97-106.

Peltoniemi, M. \& Vuori, E., 2008, 'Competitive intelligence as a driver of co-evolution within an enterprise population', Journal of Competitive Intelligence and within an enterprise po
Management 4(3), 50-62.

Prescott, J.E., 1999, 'The evolution of competitive intelligence: designing a process for action', APMP, 37-52.

Sawka, K. \& Hohhof, B., 2008, Starting a competitive intelligent function, Competitive Intelligence Foundation, Alexandria.

Shi, Z., 2011, 'Foundations of intelligence science', International Journal of Intelligence Science 1(1), 8-16. http://dx.doi.org/10.4236/ijis.2011.11002

Shi, G., Mou, X. \& Wan, X., 2009, 'Designing a network acquisition system of competitive intelligence', paper presented at the 8th IEEE International
Conference on Dependable, Autonomic and Secure Computing, Chengdu, China, Conference on Dependable, Autonomic and Secure Computing,
12-14th December. http://dx.doi.org/10.1109/DASC.2009.61

Strauss, A.C. \& Du Toit, A.S.A., 2010, 'Competitive intelligence skills needed to enhance South Africa's competitiveness', Aslib Proceedings: New Information Perspective 62(3), 302-320. http://dx.doi.org/10.1108/00012531011046925

Strategic and Competitive Intelligence Professionals (SCIP), Strategic and Competitive Intelligence Professionals, viewed 15 August 2012, from http://www.scip.org/

Venter, P. \& Tustin, D., 2009, 'The availability and use of competitive and business intelligence in South African business organisations', Southern African Business Review 13(2), 88-117.

Viviers, W., Saayman, A. \& Muller, M., 2005, 'Enhancing a competitive intelligence culture in South Africa', International Journal of Social Economics 32(7), 576-589. http://dx.doi.org/10.1108/03068290510601117

Weiss, A. \& Naylor, E., 2010, 'Competitive Intelligence: How Independent Information Professionals', American Society for Information Science and Technology 37(1) 30-34. http://dx.doi.org/10.1002/bult.2010.1720370114

Wright, S. \& Calof, J.L., 2006, 'The quest for competitive, business and marketing intelligence: a country comparison of current practices', European Journal of Marketing 40(5/6), 453-465. http://dx.doi.org/10.1108/03090560610657787

Wright, S., Eid, E.R. \& Fleisher, C.S., 2009, 'Competitive intelligence in practice: empirical evidence from the UK retail banking sector', Journal of Marketing Management 25(9/10), 941-964. http://dx.doi.org/10.1362/026725709X479318 